\title{
Coulomb Oscillations and Hall Effect in Quasi-2D Graphite Quantum Dots
}

\author{
J. Scott Bunch, Yuval Yaish, Markus Brink, Kirill Bolotin, and Paul L. McEuen*
}

Laboratory of Atomic and Solid State Physics, Cornell University, Ithaca, New York 14853

Received November 15, 2004

\begin{abstract}
We perform low-temperature electrical transport measurements on gated, quasi-2D graphite quantum dots. In devices with low contact resistances, we use longitudinal and Hall resistances to extract carrier densities of $9.2-13 \times 10^{12} \mathrm{~cm}^{-2}$ and mobilities of $200-1900 \mathrm{~cm} / \mathrm{V} \cdot \mathrm{s}$. In devices with high resistance contacts, we observe Coulomb blockade phenomena and infer the charging energies and capacitive couplings. These experiments demonstrate that electrons in mesoscopic graphite pieces are delocalized over nearly the whole graphite piece down to low temperatures.
\end{abstract}

Graphite, many stacked layers of graphene sheets separated by $0.3 \mathrm{~nm}$ and held together by weak van der Waals forces, is a low carrier density, high purity semimetal. ${ }^{1}$ The discovery of carbon nanotubes, which are rolled up graphene sheets, has brought renewed interest in this material. ${ }^{2}$ The remarkable electronic properties of nanotubes are a direct consequence of the peculiar band structure of graphene, a zero band gap semiconductor with two linearly dispersing bands that touch at the corners of the first Brillouin zone. ${ }^{3}$ Bulk graphite has been studied for decades, ${ }^{1,4-7}$ but there has been no work done on thin mesoscopic samples.

One exciting possibility is the creation of graphite quantum dots: micron scale, nanometer-thick graphite layers on an insulating substrate with patterned metallic contacts. Quantum dots have previously been made from GaAs heterostructures ${ }^{8}$ small metal grains, ${ }^{9}$ carbon nanotubes,,${ }^{10-12}$ single molecules, ${ }^{13,14}$ and many other materials, but graphite's layered structure and unusual electronic spectrum make it a promising new material for quantum dot studies. Devices with low resistance contacts would allow the basic transport parameters of the material to be determined, while those with high contact resistances $\left(R_{\mathrm{c}} \geq h / 2 e^{2}=13 \mathrm{k} \Omega\right)$ would be in the Coulomb blockade regime, where the addition and excitation spectrum of electrons could be measured.

Experiments described in this letter demonstrate two methods to wire up mesoscopic graphite pieces. Devices with low contact resistances at room temperature ("open dots") maintain their low resistance to low temperatures, and fourprobe measurements are made to extract the Hall and longitudinal resistivity of the graphite. Those with high contact resistances at room temperature ("closed dots") show Coulomb blockade oscillations at low temperatures.

The devices are fabricated as follows. Natural graphite flakes (Asbury Carbons grade 3061) are sonicated in di- chlorobenzene solution for approximately $5 \mathrm{~min}$. A drop of the solution is placed onto a degenerately doped Si wafer with a $200 \mathrm{~nm}$ thermally grown oxide. The chip is then rinsed with isopropyl alcohol and dried with nitrogen. This leaves a dispersion of graphite pieces ranging in thickness from several hundred nanometers to as small as a few nanometers (see Figure 1). We use two separate methods to wire up the graphite pieces. In the "designed electrode" method, an AFM is employed to locate thin pieces with respect to predefined alignment marks and then electron beam lithography is used to define multiple (two to six) electrodes to the piece (Figure 1b). After lithography, $50 \mathrm{~nm}$ of Pd is evaporated followed by liftoff. The resulting quasi-2D graphite quantum dots have typical lateral dimensions of approximately $1 \mu \mathrm{m}$ and vary in thickness from a few to tens of nanometers.

In the "random electrode" method, graphite is dispersed as described above and a series of electrodes with a separation of $1-2 \mu \mathrm{m}$ are defined by photolithography and evaporating $5 \mathrm{~nm}$ of $\mathrm{Cr}$ and $50 \mathrm{~nm} \mathrm{Au}$ (Figure 1d and 1e). The resistance of each pair of electrodes is measured to determine if a graphite piece was contacted. It has the advantage of being quick but lacks the control and flexibility of the designed electrode method.

The devices were measured at room temperature in a field effect geometry with a bias voltage of $10 \mathrm{mV}$ applied between source and drain electrodes. The back gate voltage $V_{\mathrm{g}}$ was varied from $10 \mathrm{~V}$ to $-10 \mathrm{~V}$ at room temperature. As shown in Figure 2, the two-point resistance of most of the devices was between 2 and $10 \mathrm{k} \Omega$, with a few $>100 \mathrm{k} \Omega$. The conductance was relatively independent of $V_{\mathrm{g}}$, but some samples showed a few percent decrease in the conductance with positive $V_{\mathrm{g}}$.

Low temperature measurements on the devices were performed at $1.5 \mathrm{~K}$ in an Oxford variable temperature insert 


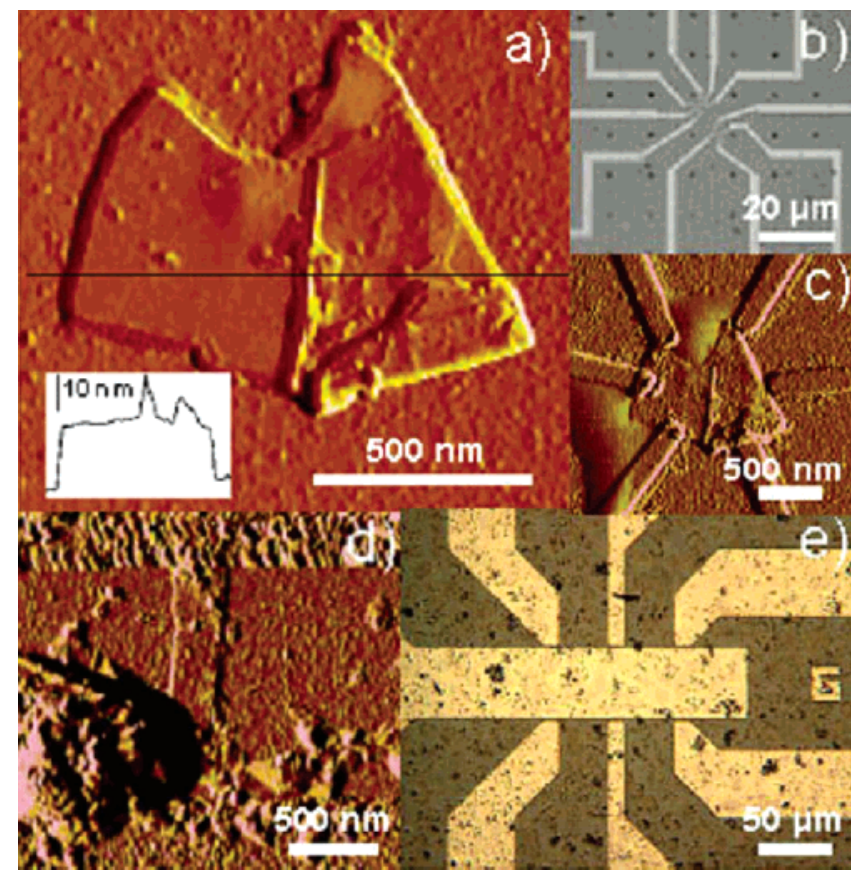

Figure 1. (a) AFM image of a graphite piece with a height of 18 nm dispersed onto a $\mathrm{SiO}_{2} / \mathrm{Si}$ wafer. (inset) Line trace showing the height. (b) Optical image of electrodes fabricated by electron beam lithography. When a desired graphite piece is located in AFM, its position with respect to the predefined alignment marks visible in the image is determined. Electron beam lithography and liftoff is then used to define the electrodes. (c) AFM image of six electrodes defined by electron beam lithography contacting an $18 \mathrm{~nm}$ thick graphite dot (designed electrode). (d) AFM image of two electrodes contacting a $5 \mathrm{~nm}$ thick graphite dot (random electrode). (e) Optical image of a set of electrodes defined by photolithography over randomly dispersed graphite (random electrode).

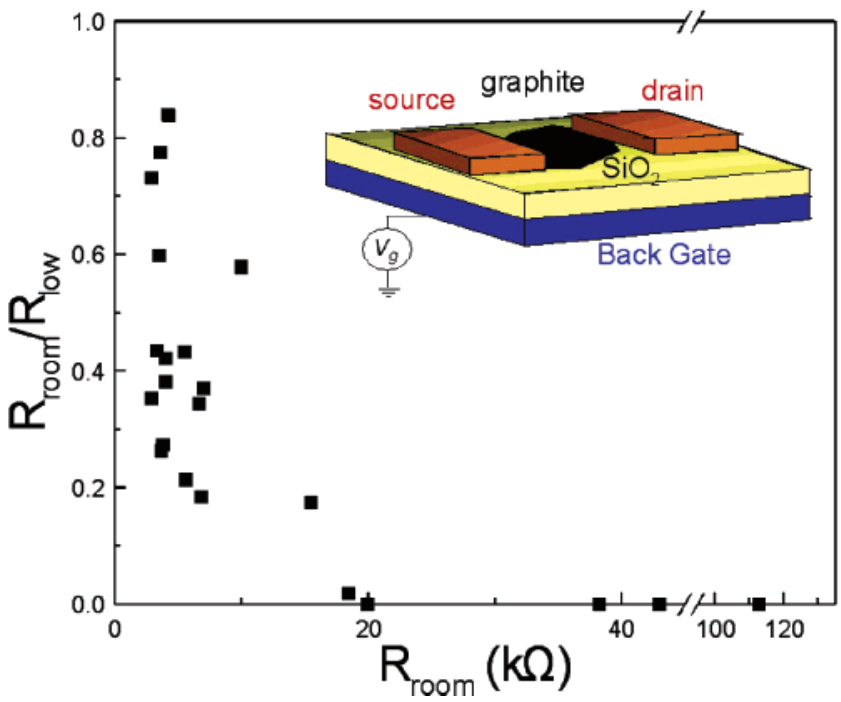

Figure 2. Scatter plot of the ratio of the low $(T \sim 100 \mathrm{mK})$ to room temperature two-point resistance versus the room-temperature two-point resistance for all the devices for which there is lowtemperature data. (inset) Schematic of the device layout. The graphite is in a field effect transistor geometry with a $200 \mathrm{~nm}$ gate oxide. Source and drain electrodes are patterned on top.

(VTI) cryostat or $100 \mathrm{mK}$ in an Oxford dilution refrigerator. The devices with low resistances at room temperature (open dots) displayed only a small increase in their resistance upon

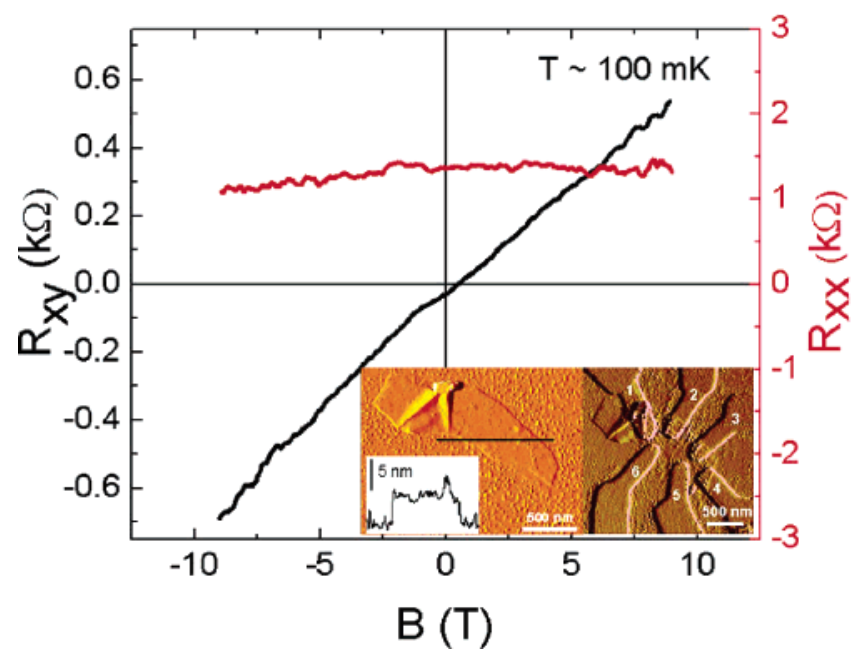

Figure 3. Longitudinal and Hall resistance measured as a function of magnetic field at $100 \mathrm{mK}$ for the $5 \mathrm{~nm}$ thick graphite dot shown in the insets. The Hall resistance, $R_{x y}$, was determined using standard lock-in techniques by applying a $43 \mathrm{nA}$ excitation current between electrodes 2 and 6 and measuring the voltage drop between electrodes 1 and 4 . The longitudinal resistance, $R_{x x}$, was determined by measuring the voltage drop between electrodes 5 and 6 while an excitation current of $10 \mathrm{nA}$ was passed between electrodes 1 and 4 . The slope of $R_{x y}$ versus $B$ (black line) corresponds to a total density of $9.2 \times 10^{12} \mathrm{~cm}^{-2}$. The longitudinal resistance (red line) shows only weak fluctuations as a function of $B$. (inset a) AFM image of a graphite piece with a height of $5 \mathrm{~nm}$ and its corresponding line trace. (inset b) The graphite piece shown in inset (a) with electrodes patterned on top using the designed electrode method.

cooling, as seen in Figure 2. In such devices with multiple contacts, we performed longitudinal and Hall resistance measurements to extract the carrier density, sign of the carriers, and resistivity. Figure 3 shows data from a $5 \mathrm{~nm}$ tall dot, corresponding to approximately 15 stacked graphene sheets, measured at $\sim 100 \mathrm{mK}$ using standard AC lock-in techniques. Similar results were obtained at 4 and $1.5 \mathrm{~K}$. The Hall resistance $R_{x y}$ is approximately linear, and the longitudinal resistance $R_{x x}$ shows weak fluctuations as a function of magnetic field with little change in its average value.

To analyze these results, we make the simplifying assumption that the entire graphite piece is a uniform conductor with a single density and in-plane mobility. From the standard equation for the Hall resistance $R_{\mathrm{H}}=B / n e$, the slope of the line in Figure 3 corresponds to a density of $9.2 \times$ $10^{12} \mathrm{~cm}^{-2}$. The sign of the Hall voltage indicates that the dominant charge carriers are holes. A similar measurement on a second device with a height of $18 \mathrm{~nm}$, shown in Figure $1 \mathrm{a}$ and $1 \mathrm{c}$, gives a hole density of $1.3 \times 10^{13} \mathrm{~cm}^{-2}$.

After accounting for the geometrical factors, we infer the resistance per square, $R_{s}$, of the entire sample. For the $5 \mathrm{~nm}$ thick device at $100 \mathrm{mK}$, we have $R_{s}=3.4 \mathrm{k} \Omega$. Using the equation $\mu=1 / n e R_{s}$, we get a mobility of $\mu=200$ $\mathrm{cm}^{2} / \mathrm{V} \cdot \mathrm{s}$. A similar analysis for the sample with a thickness of $18 \mathrm{~nm}$ shown in Figure $1 \mathrm{a}$ and $1 \mathrm{c}$ at $1.5 \mathrm{~K}$ yields $R_{s}=$ $260 \Omega$ and $\mu=1900 \mathrm{~cm}^{2} / \mathrm{V} \cdot \mathrm{s}$. The inferred mobilities are significantly lower than in bulk-purified natural graphite flakes, which range from 1.5 to $130 \times 10^{4} \mathrm{~cm}^{2} / \mathrm{V} \cdot \mathrm{s}$. 
(a)

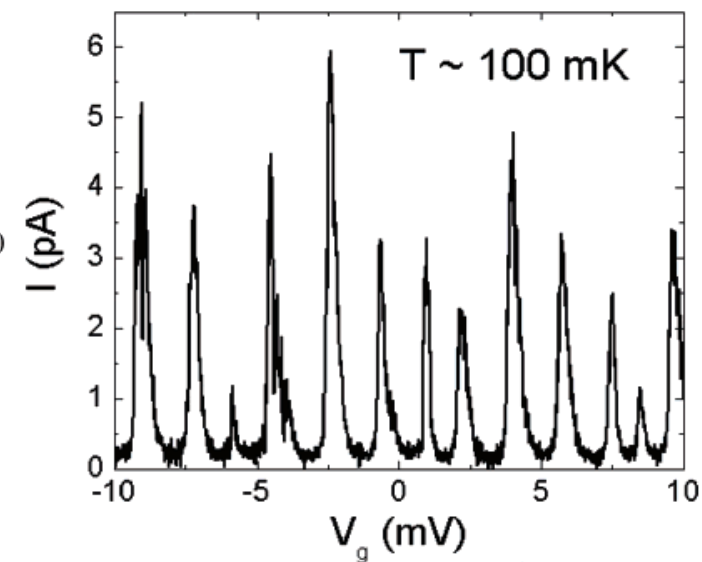

(b)

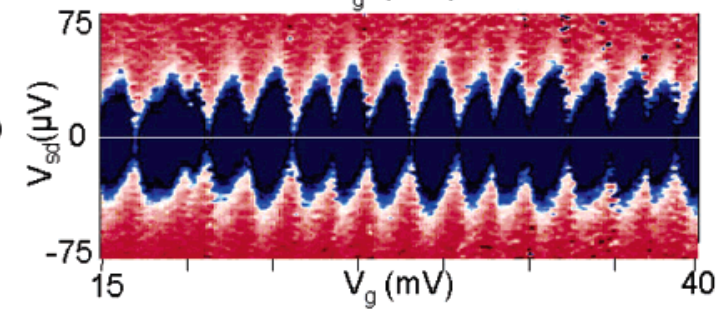

Figure 4. (a) Current as a function of gate voltage with $V_{\text {sd }}=10$ $\mu \mathrm{V}$ at $T \sim 100 \mathrm{mK}$ for a device fabricated by the random electrode method. Coulomb oscillations are observed with a period in gate voltage of $\Delta V_{\mathrm{g}}=1.5 \mathrm{mV}$. (b) The differential conductance $\mathrm{d} I / \mathrm{d} V_{\text {sd }}$ plotted as a color scale versus $V_{\text {sd }}$ and $V_{\mathrm{g}}$. Blue signifies low conductance and red high conductance. The charging energy of the quantum dot is equal to the maximum height of the diamonds: $\Delta V_{\mathrm{sd}}=0.06 \mathrm{mV}$. The center-to-center spacing between the diamonds is the Coulomb oscillation period $\Delta V_{\mathrm{g}}=1.5 \mathrm{mV}$.

We can use a gate to vary the carrier density in the graphite quantum dot. We assume the capacitance to the gate is that of a parallel plate capacitor, $C_{\mathrm{g}}=\epsilon_{\mathrm{o}} \epsilon A / d$, where $d=200$ $\mathrm{nm}$ is the thickness of the $\mathrm{SiO}_{2}, \epsilon_{\mathrm{o}}$ is the permittivity of free space, $\epsilon$ is the dielectric constant of $\mathrm{SiO}_{2}$, and $A$ is the area of the device. This gives a capacitance per area of $C_{\mathrm{g}}{ }^{\prime}=1.8$ $\times 10^{-8} \mathrm{~F} / \mathrm{cm}^{2}$, implying that $10 \mathrm{~V}$ applied to the back gate results in a change of density of $1 \times 10^{12}$ holes $/ \mathrm{cm}^{2}$. This is only a small fraction of the total density in even the thinnest samples studied. Nevertheless, it is consistent with a small decrease in conductance observed in many samples at room temperature; the holes are slightly depleted by the gate. At low temperatures, any such changes are obscured by reproducible fluctuations in the conductance as a function of $V_{\mathrm{g}}$.

Devices with room-temperature two-point resistances greater than $20 \mathrm{k} \Omega$ (closed dots) show Coulomb blockade at low temperatures. Data from a device fabricated using the random electrode method is shown in Figure 4. At $T=$ $100 \mathrm{mK}$, the conductance exhibits well-defined Coulomb blockade oscillations with a period in gate voltage of $\Delta V_{\mathrm{g}}$ $=1.5 \mathrm{mV}$. A plot of $\mathrm{d} I / \mathrm{d} V_{\mathrm{sd}}$ vs $V_{\mathrm{g}}$ and $V_{\mathrm{sd}}$ is shown in Figure 4. The maximum voltage that could be applied and still be in the blockade regime is $\Delta V_{\mathrm{sd}}=0.06 \mathrm{mV}$.

A device made by the designed electrode method is shown in Figure 5. The thickness of the device is $6 \mathrm{~nm}$, corresponding to 18 sheets. The Coulomb blockade oscillations have a period of $\Delta V_{\mathrm{g}}=11 \mathrm{mV}$ and a maximum blockade (a)
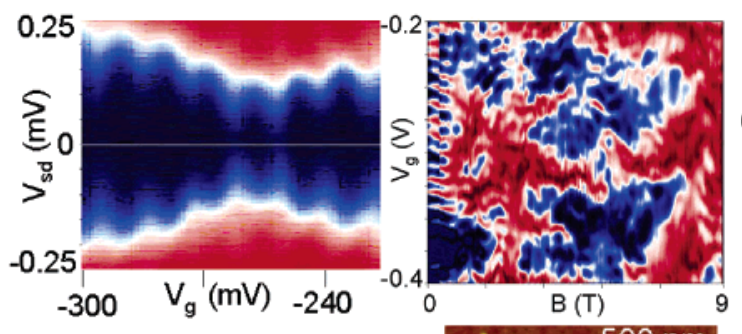

(c)

(b)
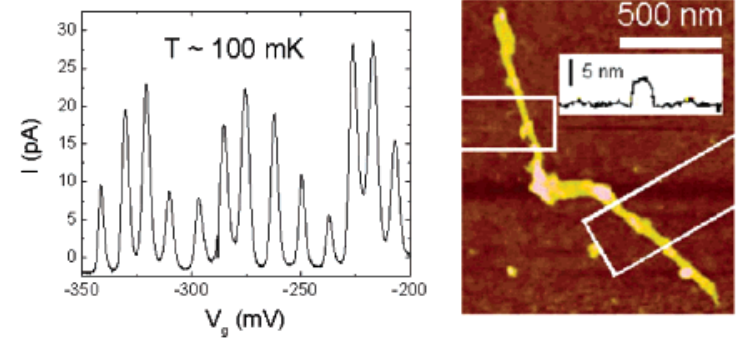

(d)

Figure 5. (a) The differential conductance $\mathrm{d} I / \mathrm{d} V_{\text {sd }}$ plotted as a color scale versus $V_{\text {sd }}$ and $V_{\mathrm{g}}$ for the device shown in (d) at $T \sim 100$ $\mathrm{mK}$. The charging energy is $\Delta V_{\mathrm{sd}}=0.3 \mathrm{mV}$ and the gate voltage period is $\Delta V_{\mathrm{g}}=11 \mathrm{mV}$. (b) Current as a function of gate voltage with $V_{\text {sd }}=10 \mu \mathrm{V}$ at $T \sim 100 \mathrm{mK}$ for the device shown in (d). Coulomb blockade oscillations are seen with a period of $\Delta V_{\mathrm{g}}=$ $11 \mathrm{mV}$. (c) Current as a function of gate voltage versus magnetic field with $V_{\text {sd }}=10 \mu \mathrm{V}$ at $T \sim 100 \mathrm{mK}$ for the device shown in (d). (d) AFM image of a device fabricated using the designed electrode method. The white rectangular outlines show the position and size of the electrodes that were evaporated on the device. The total area of the graphite piece is $0.12 \mu \mathrm{m}^{2}$ and the area under the source and drain electrodes is $0.013 \mu \mathrm{m}^{2}$ and $0.015 \mu \mathrm{m}^{2}$, respectively. The area of the graphite piece between the source and drain electrodes is $0.05 \mu \mathrm{m}^{2}$. (inset) Line trace showing the $6 \mathrm{~nm}$ height of the graphite.

voltage of $\Delta V_{\mathrm{sd}}=0.3 \mathrm{mV}$. A third device fabricated by the random electrode method shown in Figure 1d has a height of $5 \mathrm{~nm}$ and shows Coulomb oscillations with a period in gate voltage of $\Delta V_{\mathrm{g}}=1.3 \mathrm{mV}$.

To describe these results, we use the semiclassical theory of the Coulomb blockade. ${ }^{15}$ The period of the Coulomb oscillations in gate voltage is given by: $\Delta V_{\mathrm{g}}=e / C_{\mathrm{g}}$ and, using the previous expression for $C_{\mathrm{g}}$, we can approximate the area of the graphite quantum dot. For the device in Figure 5 with $\Delta V_{\mathrm{g}}=11 \mathrm{mV}$, the expected area of dot is $A=0.08$ $\mu \mathrm{m}^{2}$. The measured total area of the graphite piece shown is $0.12 \mu \mathrm{m}^{2}$ while the area between the electrodes is $0.05 \mu \mathrm{m}^{2}$. This demonstrates that nearly the entire graphite piece is serving as a single quantum dot and it likely extends beyond the electrodes. For the device shown in Figure 1d, the measured gate voltage period is $\Delta V_{\mathrm{g}}=1.3 \mathrm{mV}$, which corresponds to a quantum dot with $A=0.70 \mu \mathrm{m}^{2}$. The area between the electrodes is $0.45 \mu \mathrm{m}^{2}$ again implying that the dot extends into the graphite piece lying under the electrodes.

The charging energy for the dot is determined by its total capacitance $C$ and is equal to the maximum blockade voltage observed: $e / C=\Delta V_{\mathrm{sd}}$. Notably, for our graphite quantum dots, the ratio of the charging energy to the gate voltage period is small: $\alpha=(e / C) / \Delta V_{\mathrm{g}}=C_{\mathrm{g}} /\left(C_{\mathrm{s}}+C_{\mathrm{d}}+C_{\mathrm{g}}\right) \ll 1$, where $C_{\mathrm{s}}$ and $C_{\mathrm{d}}$ are the capacitances to the source and drain electrodes. The devices shown in Figure 4 and Figure 5 have $\alpha=1 / 25$ and $1 / 40$, respectively. Such small values imply 
that the graphite pieces have a much greater capacitive coupling to the source and drain electrodes than to the gate.

We can estimate the source and drain capacitance per unit area $C_{\mathrm{s}, \mathrm{d}}$ ' using the measured charging energies and the area of the electrodes over the graphite. For the device shown in Figure 5, this yields a capacitance per unit area of $C_{\mathrm{s}, \mathrm{d}}{ }^{\prime}=2$ $\times 10^{-6} \mathrm{~F} / \mathrm{cm}^{2}$. Using the parallel plate capacitor equation, $C_{\mathrm{s}, \mathrm{d}}{ }^{\prime}=\epsilon_{\mathrm{o}} \epsilon_{\mathrm{r}} / d$ yields $d / \epsilon_{\mathrm{r}}=0.5 \mathrm{~nm}$. This is consistent with a very thin tunnel barrier between the electrode and the graphite. The origin and nature of this barrier is unknown. Since it is only present in the few samples that show Coulomb blockade oscillations (closed dots), it is likely the result of a contamination layer between the metal and the graphite.

We also examined the magnetic field dependence of the Coulomb blockade oscillations (Figure 5c). The closed dot at $B=0 \mathrm{~T}$ has well-defined oscillations. As the magnetic field is increased, the peaks evolve in a complicated fashion. Most notably, the oscillations no longer go to zero, suggesting that the dot becomes more open. The open dot shows complex fluctuations in the peak positions as a function of magnetic field. Other devices showed similar transitions from closed to more open dots along with changes in the peak positions as a function of field. The origin of these effects is under investigation.

In conclusion, we fabricated and measured quasi 2-D graphite quantum dots. In devices with good contacts (open dots), we performed resistivity measurements and extracted carrier densities of $9.2-13 \times 10^{12} \mathrm{~cm}^{-2}$ and mobilities of $200-1900 \mathrm{~cm}^{2} / \mathrm{V} \cdot \mathrm{s}$. In the case of tunnel contacts (closed dots), we observed Coulomb charging phenomena and inferred the gate and source-drain capacitances. Future studies will investigate the nature and role of interlayer coupling between the sheets and explore the single-particle energy level spectrum and the effects of a magnetic field. Studies on devices with a variety of thicknesses and improved control over the contacts will help address these issues.
Note Added: During preparation of this manuscript, we became aware of three other groups ${ }^{16-18}$ reporting transport measurements of thin graphite layers that are related to our measurements of open dots (Figure 3).

\section{References}

(1) Kelly, B. T. Physics of graphite; Applied Science: London, Englewood, N.J., 1981; pp 267-361.

(2) McEuen, P. L. Phys. World 2000, 13(6), 31-36.

(3) Wallace, P. R. Phys. Rev. 1947, 71(9), 622-634.

(4) Luk'yanchuk, I. A.; Kopelevich, Y. Phys. Rev. Lett. 2004, 93(16), 166402.

(5) Du, X.; Tsai, S.-W.; Maslov, D. L.; Hebard, A. F., 2004, arXiv: cond$\mathrm{mat} / 0404725$

(6) Tokumoto, T.; Jobiliong, E.; Choi, E. S.; Oshima, Y.; Brooks, J. S. Solid State Commun. 2004, 129(9), 599-604.

(7) Soule, D. E. Phys. Rev. 1958, 112(3), 698-707.

(8) Kouwenhoven, L. P.; Marcus, C. M.; McEuen, P. L.; Tarucha, S.; Westervelt, R. M.; Wingreen, N. S. Electron transport in quantum dots. In Mesoscopic Electron Transport; Sohn, L. L., Kouwenhoven, L. P., Schoen, G., Eds.; Plenum: New York, London, 1997; pp 105214.

(9) Ralph, D. C.; Black, C. T.; Tinkham, M. Phys. Rev. Lett. 1997, 78(21), 4087-4090.

(10) Bockrath, M.; Cobden, D. H.; McEuen, P. L.; Chopra, N. G.; Zettl, A.; Thess, A.; Smalley, R. E. Science 1997, 275(5308), 1922-1925.

(11) Tans, S. J.; Devoret, M. H.; Dai, H. J.; Thess, A.; Smalley, R. E.; Geerligs, L. J.; Dekker, C. Nature 1997, 386(6624), 474-477.

(12) Buitelaar, M. R.; Bachtold, A.; Nussbaumer, T.; Iqbal, M.; Schonenberger, C. Phys. Rev. Lett. 2002, 88(15), 156801.

(13) Park, J. W.; Pasupathy, A. N.; Goldsmith, J. I.; Soldatov, A. V.; Chang, C.; Yaish, Y.; Sethna, J. P.; Abruna, H. D.; Ralph, D. C.; McEuen, P. L. Thin Solid Films 2003, 438, 457-461.

(14) Liang, W. J.; Shores, M. P.; Bockrath, M.; Long, J. R.; Park, H. Nature 2002, 417(6890), 725-729.

(15) Beenakker, C. W. J. Phys. Rev. B 1991, 44(4), 1646-1656.

(16) Berger, C.; Song, Z.; Li, T.; Li, X.; Ogbazghi, A. Y.; Feng, R.; Dai, Z.; Marchenkov, A. N.; Conrad, E. H.; First, P. N.; de Heer, W. A., 2004, arXiv: cond-mat/0410240.

(17) Zhang, Y.; Small, J. P.; Amori, M. E. S.; Kim, P., 2004, arXiv: condmat/0410315

(18) Novoselov, K. S.; Geim, A. K.; Morozov, S. V.; Jiang, D.; Zhang, Y.; Dubonos, S. V.; Grigorieva, I. V.; Firsov, A. A. Science 2004, 306(5696), 666-669.

NL048111+ 